\title{
EFEITO IN VITRO DA JOSAMICINA EM CEPAS DE Rhodocaccus equi ISOLADAS DE AFECÇÕES PULMONARES EM POTROS
}

\author{
MÁRCIO GARCIA RIBEIRO ' \\ ALDO SANTANA DE CARVALHO FILHO ${ }^{3}$ \\ JANE MEGID \\ FERNANDO JOSÉ PAGANINI LISTONI \\ JAIME GALVÃO DIAS JÚNIOR 4
}

\begin{abstract}
RIBEIRO, M. C.; CARVALHO FILHO, A. S. DE; MEGID, J.; LISTONI, F. J. P.; DIAS JúNJOR. J. G. Efeito in vitro da josamicina em cepas de Rhodococcus equi isoladas de afecções pulmonares em potros. Semina: Ci. Agrárias. Londrina, v, 21, n. 1, p. 3-8, mar, 2DDQ.
\end{abstract}

RESUMO: Procedeu-se o teste de sensibilidade microbiana in vitro pelo teste de difusão com discos em 31 cepas de Rhodococcus equi. isoladas de afecções pulmonares cm potros. frente a 20 antimicrobianos. Os maioires índices de sensibilidade de $R$. equi foram constatados para entromicina (100,0 \%), níampicina (96.8\% $\}_{t}$ ncomicina (93. 6 \%). josamicina (90.4\%) e gentamicina (90A \%). Os maiores índices de resistência do agente foram verificados para cefalexina (100,0\%), lincomicina (100.0\%), cefalotina (96.8 \%), oxacilino (96,8 \%), penicilina G (96,3\%), amoxicilina (90.3\%) e sulfametoxazol/trimetoprim (83,8 \%). A alta sensibilidade das cepas de $R$. equi para a josamicina, sugere a possibilidade de utilização da droga como alternativa no tratamento da rodococose em potros.

PALAVRAS-CHAVE: Rhodococcus equi, sensibilidade microbiana, tratamento.

\section{INTRODUÇÃ O}

Rhodococcus equi ( $R$ - equi) é reconhecido como organismo intracelular facultativo, ubíquo, de caráter oportunista, refratário à terapia antimicrobiana convencional, relacionado a diferentes afecções no homem e em animais (Barton \& Hughes, 1980; Linder, 1997).

A primeira descrição de $R$. equi, em animais, foi realizada por Magnussorn, em 1923, na Suíça, em eqüino apresentando pneumonia granulomatosa crônica (Macmussorn, 1923 apud Prescott, 1991). Estudos subseqüentes, tem identificado o microrganismo como o agente causal mais importante de processos respiratórios em potros (Giguére \& Prescott, 1997).

Além de distúrbios respiratórios em eqüinos jovens, $R$. equí tem sido associado à outras manifestações clínicas em diferentes espécies de interesse econômico e em animais silvestres, destacando-se quadros de enterite, linfadenite, aborto, masite. dermatite e piometra (Barton \&. Hughes, 1980; Prescolt 1991).
No Brasil, $R$. equi também tem sido considerado como importante agente de pneumonia de caráter abscedante em potros, caracterizado pelo difícil tratamento (Corrêa \& Corroa. 1992; Langoni 1994).

No homem, a rodococose é apontada como doença emergente, relacionada à indivíduos severamente comprometidos, incluindo convalescentes de transplantes, alcoólatras, pacientes com neoplasias malignas ou sob terapia imunossupressiva (Doig et ai., 1991; Prescott, 1991; Linder, 1997). Em anos recentes, é crescente o numero de casos de infecções por $R$. equi em pessoas acometidas pela síndrome da imunodeficiência adquirida-AIDS (Drancovrt et al., 1992; Donisi et al., 1996; Mosser 8, Hondalus, 1996). ou mesmo em indivíduos saudáveis, especialmente crianças (Macgowan \& Mangano, 1991). A transmissão do $R$ - equi para o homem, tem sido atribuída ao contato recente com animais, principalmente da espécie eqüina (Doig et al., 1991), ou á exposição ao agente no ambiente, geralmente secundaria à lesões transcutâneas (Proscoutt, 1991).

Professores da Disciplina de Enfermidades Infecciosas cios Ammais. Departamento do Higiene Veterinária c Saúde Pública-DHVSP, Faculdade de Medicina Veterinariae Zootecnia (FMVZ)-UNESP Fone (014) 6802-5270, Fax 6802.6075, CP-560, 18618-000, BotucatuSP

Acadêmico de Medicina veterinária-FMVZ-UNESP Botucatu-SP. Bolsista de Iniciação Cientifica. Programa PIBIC-CNPq-UNESP Auxiliar técnico-DHVSP-FM VZ-UNESP - Botucatu-SP.

Residense na área de Enfermidades Infecciosas dos Animais-OHVSr^-FMVZ-UMESPyBotuCíitu-SP 
Determinados fatores de virulência e mecanismos de evasão do sistema imune possibilitam ao $R$. equi multiplicar-se no interior de fagócitos (neutrófilos e macrófagos), dificultando o estabelecimento de resposta imune adequada, induzindo lesões do tipo piogranulomatosa (Hines et al., 1997), levando, consequentemente, a processos de difícil resolução tecidual e refratários aos tratamentos com antimícrobianos convencionais (Vivrette, 1992),

Os mecanismos que permitem a viabilidade do $R$. equi em fagócitos, condicionam a efetividade da terapia ao uso de drogas lipofílicas, como a eritromicina e a rifampicina. que possuem capacidade de penetração em neutrófilos, macrófagos (Hillidge, 1986) e em material caseoso (Giguère \& Prescott, 1997).

Estudos conduzidos na investigação da sensibilidade microbiana de $R$. equi., isolado de diferentes afecções em animais, apontam que as drogas de maior efetividade "in vitro", incluem antimícrobianos do grupo dos rnacrolídeos (eritromicina), quinolonas (enrofloxacina, ciprofloxacina), aminoglicosideos (gentamícina, amicacina, neomicina) e rifampicina, além de associações entre a rifampicina e eritromicina ou ácido clavulânico e ticarcilina (Woolcock \& Multimer, 1930; Hillidge, 1987; Sparks et al., 1988; Prescott, 1991; Giguère \& Prescott, 1997; Pronost et ai., 1998). A associação entre rifampicina e eritromicina apresenta efeito sinêrgico "in vitro" e "in vivo" em cepas de $R$. equi (Prescott \& Nicholson, 1984), constituindo-se, atualmente, no tratamento de eleição da rodococose em potros (Prescott, 1991; Pronost et al., 1998).

As limitações no sucesso terapêutico direcionado às infecções por $R$. equi, fundamentado no emprego de antimícrobianos, tanto no homem quanto em animais, têm motivado estudos de sensibilidade in vitro do agente, ensaios com novas drogas e suas associações, visando maior efetividade na terapia da rodococose (Hillidge. 1987; Harvey \& Sunstrun, 1991 ; Drancourt et al., 1992; Giguère \& Prescott, 1997).

Diferentes antimícrobianos vêm sendo investigados alternativamente (in vitro, in vivo ou em animais de laboratório) na terapia da rodococose, incluindo amicacina, vancomicina, clindamicina, lincomicina, penicilína/gentamicina, ticarcilína/ácido clavulônico, entre outros. Entretanto, a maioria destas drogas têm apresentado resultados controversos ou insatisfatórios em grande parte destes estudos direcionados à terapia antimicrobiana alternativa do $R$, equi (Sweeney et al., 1987; Sparks et al., 1989; Prescott, 1991; Nordmann et al., 1992). A reduzida efetividade destas drogas decorre, provavelmente, da resistência do agente a determinados grupos de antimícrobianos (natural ou adquirida, do alto custo destes medicamentos, das reações locais após a aplicação de produtos via intramuscular, das reações sistêmicas em animais tratados por período prolongado, ou mesmo, por descontinuidade do tratamento (Doig et al., 1991; Prescott, 1991; Giguère \& Prescott, 1997).

A josamicina constitui-se em um antrmicrobiano do grupo dos macrolideos, originada do Streptomyces narbonensis (Tavares, 1996a), indicada, originalmente, para o tratamento (via oral) de afecções pulmonares de suínos e aves.

O princípio ativo da josamicina não sofre inativação gástrica, apresenta alta absorção intestinal, baixa toxicidade, rápida concentração sèrica e pulmonar, e ação bactericida. especialmente para microrganismos grampositivos (Tavares, $1996 \mathrm{a}$ ), mediante sua ação no ribossoma, interferindo na síntese protéica bacteriana. A droga possui propriedades de estimulação inespecífica da resposta imune, incluindo quimiotaxia de neutrófilos, macrófagos e células NK (natural killer). além da penetração nestas células inflamatórias. favorecendo a sua distribuição no organismo (Virbac, 1999).

Neste sentido, o presente estudo objetivou investigar a sensibilidade in vitro de cepas de $\mathrm{R}$. equi, isoladas de afecções pulmonares em potros, frente ã josamicina.

\section{MATERIAL E MÉTODO}

Foram examinadas 29 cepas de $R$. equi, isoladas de afecções pulmonares em potros, provenientes do material de rotina do Laboratório de Diagnóstico Microbiológico de Enfermidades Infecciosas dos Animais, do Departamento de Higiene Veterinária e Saúde Pública, da Faculdade de Medicina Veterinária e Zootecnia-UNESP/ Campus de Botucatu-SP; do Laboratório de Bacteriologia do Departamento de Medicina Veterinária Preventiva da Universidade Federal de Santa Maria-RS e da Coleção de Culturas do Instituto Adolfo Lutz de São Paulo-SP.

Adicionalmente, foram utilizadas como controle duas cepas de $R$. equi (ATCG 33.701-e ATCC 33.701 $+)$, cedidas pelo Instituto de Biociências da UNESP/ Botucatu-SP, provenientes da Faculdade de

"Josamicina - Alplucine* - Virbac do Brasi 
Medicina Veterinária de Ontário (Guelph), Canadá; perfazendo a avaliação de 31 cepas do agente.

Todas as cepas de $R$. equi foram submetidas ao Leste de sensibilidade microbiana - método de difusão com discos - (Bauer et al., 1966), utilizando os seguintes antimicrobianos: amicacina (30 $\mathrm{mcg})$, amoxilina $(10 \mathrm{mcg})$. ampicilina (10 $\mathrm{mcg})$, canamicina $(3 \mathrm{Q} \mathrm{mcg})$. cefalexina $(30 \mathrm{mcg})$. cefalotina $(30 \mathrm{mcg})$, ciprofloxacina $(5 \mathrm{mcg})$. cloronfenicol (30 mcg). enrofloxacina (5 mcg). entremicina (15 mcg). estrepiomicina (10 mcg), gentamicina (10 mcg), josamicina (150 mcg), lincomicina ( $2 \mathrm{mcg}$ ), neomicina $(30 \mathrm{mcg})$, oxacilina (5 mcg), penicilina G (10 UI), rifampicina (5 mcg). sulfametoxazol/trimeloprim (25 $\mathrm{mcg})$ e tetraciclina $(30 \mathrm{mcg})$.

\section{RESULTADOS}

A Tabela 1 apresenta a sensibilidade microbiana de cepas de $R$. equi. isoladas de afecções pulmonares em potros, utilizando-se 20 diferentes antimicrobianos. Verificou-se que os maiores índices de sensibilidade do R. equi foram constatados para eritromicina $(100,0 \%)$, rifampicina $(96,8 \%)$, neomicina $(93,6 \%)$, josamicina $(90,4 \%)$ e gentamicina $(00,4 \%)$. Em contraste, os maiores índices de resistência do agente foram verificados para cefalexina $(100,0$

lincomicina $(100,0 \%)$, cefalotina $(95,3 \%)$, oxacilina $(96,8 \%)$, penicilina G $(96,8 \%)$, amoxicilina $(90,3$ $\%)$ e suífametoxazol/trimetoprim $(83,8 \%)$.

Tabela 1 - Sensibilidade microbiana. na prova de difusão com discos, em 31 cepas de Rhodococcus equi isoladas de afecções pulmonares em potros.

Antimicrobianos

\begin{tabular}{|c|c|c|c|}
\hline & $\mathrm{s}(\%)$ & $1(\%)$ & $\mathrm{R}(\%)$ \\
\hline Amicacina $\{30 \mathrm{mog}\rangle$ & $18(58,0)$ & $9(29,0)$ & $4(13,0)$ \\
\hline Amoxicilina $(10 \mathrm{mog})$ & $3(9,7)$ & $0(-)$ & $28(90,3)$ \\
\hline Ampicilina $(10 \mathrm{mcg}$ ) & $4(13,0)$ & $4(13,0)$ & $23(74,0)$ \\
\hline Canamicina (30 mog) & $0(-)$ & $9(20,0)$ & $22(7-1,0)$ \\
\hline Cefalexina $(30 \mathrm{mcg})$ & $0(--)$ & $0(-)$ & $31(100,0)$ \\
\hline Cefalotina (30 mcg) & $1\{3,2\}$ & $0(--)$ & $30(06.8)$ \\
\hline Ciprofoxacina (5 mog) & $20(64,5)$ & $10(32,3)$ & $1(3,2)$ \\
\hline Clonanfenicol (30 mog) & $6(19,3)$ & $19(61,4)$ & $6(19,3)$ \\
\hline Enrofloxacina $(5 \mathrm{mcg})$ & $11\{35,5\}$ & $14(45,2)$ & $6(19,3)$ \\
\hline Eritromicina $(15 \mathrm{mcg})$ & $31(100.0)$ & $0(-)$ & $0(-)$ \\
\hline Estieptomicina (10 mog) & $17(54,8)$ & $7(22,6)$ & $7(22,6)$ \\
\hline Gentanicina (10 mog) & $28(90,4)$ & $2\langle 6,4\}$ & $1\{3,2\}$ \\
\hline Josamicina (150 mcg) & $28(90,4)$ & $3\{9,6)$ & $0(-)$ \\
\hline Lincomicina (2 mog) & $0(-)$ & $0(-)$ & $31(100,0)$ \\
\hline Neomicina (30 meg) & $29(93,6)$ & $0(-\infty)$ & $2(6,4)$ \\
\hline Oxacilina (5 mog) & $1(3,2)$ & $0(-)$ & $30(96,8)$ \\
\hline Penicilina $G$ (10 LA) & $1(3,2)$ & $0(-)$ & $30(96,8)$ \\
\hline Rifampicina (5 meg) & $30\{96,8)$ & $0(-)$ & $1(3,2)$ \\
\hline Sulfametoxazol / Trimetoprim (25 mog) & $0(-)$ & $5(16,2)$ & $26 \cdot(83,8)$ \\
\hline Tetraciclina ( $30 \mathrm{mco}$ ) & $2(6,4)$ & $19\{58,1\}$ & $f 1(35,5)$ \\
\hline
\end{tabular}




\section{DISCUSSÃO E CONCLUSÕES}

A elevada resistência ou sensibilidade intermediária (parcial) in vitro em grande parte das cepas de $R$. equi frente aos diferentes antimícrobianos utilizados no presente estudo (Tabela 1), com exceção de drogas como a eritromicina e a rifampicina. concorda com os achados de outros estudos de sensibilidade in vitro do agente a diferentes antibióticos e quimioterápicos (Giguère \&. Prescott, 1997; Pronost et al., 1993). Este resultado reforça a indicação da associação entre a eritromicina e a rifampicina, como drogas de primeira escolha na terapia da rodococose pulmonar em potros.

Apesar da indicação da rifampicina e eritromicina para a maioria dos casos de infecções pulmonares por R. equi em potros, outros antimícrobianos têm sido empregados de forma alternativa, em virtude de determinados fatores limitantes na manutenção da terapia da rodococose, com a associação destas drogas. Estas limitações incluem, principalmente, a resistência (simples ou adquirida) de cepas de $R$. equi, o alto custo destes medicamentos, ou a ocorrência de reações orgânicas indesejáveis em animais tratados por longo período com a rifampicina e/ou eritromicina (Hillidge, 1986 ; Prescott, 1991; Giguère \& Prescott, 1997).

Dentre as drogas investigadas como alternativas no tratamento, destaca-se o grupo das quinolonas, como a enrofloxacina e ciprofloxacina. Apesar das quinolonas apresentarem alta concentração em fagócitos como a rifampicina e eritromicina, e possuírem apresentação para administração por via oral (com a vantagem de poderem ser prescritas em intervalos de 24 horas), este grupo de drogas não apresenta, até o momento, indicação para a espécie eqüina. Adicionalmente, a administração oral de enrofloxacina por período prolongado, tem desencadeado, experimentalmente, quadros de laminite, lesões de cartilagem e articulares, além de várias cepas de $R$. equi demonstrarem altos indices de sensibilidade intermediária (Guiguère \& Prescott, 1997), resultado também obtido no presente estudo (Tabela 1), o que provavelmente tem desencorajado a sua aplicação na espécie eqüina.

Altas doses da associação trímetoprimsulfametoxazol têm sido preconizadas na terapia de afecções pulmonares em potros por $R$. equi, exclusivamente em estágios iniciais da infecção, sem evidência de marcada abscedação pulmonar (Wilson, 1992). Entretanto, esta associação tem demonstrado baixa eficiência em animais com pneumonia abscedante pronunciada, decorrente, provavelmente, da reduzida penetração destas drogas em fagócitos e em material caseoso (Giguère \& Prescott, 1997). O grande número de cepas de $R$. equi parcialmente sensíveis ou resisteníes in vitro à associação trimetoprimsuframetcazol observado no presente estudo (Tabela 1), aliado a reduzida capacidade de penetração intracelular e em processos purulentos destas drogas (Giguère \& Prescott, 1997), tendem a limitar a indicação desta associação na terapia da rodococose em potros.

A administração de penicilina $G$, via endovenosa, têm demonstrado efetividade em determinados casos de pneumonia abscedante por $R$. equi em potros (Gay et al., 1981). Em contraste, no presente estudo, verificou-se altos indices de resistência á penicilina e a derivados do anel 8 lactâmico (amoxicilina, ampicilina, cefalexina. cefalotina e oxacilina) [Tabela 1]. Estes resultados coincidem com outros ensaios de sensibilidade in vitro do $R$. equi, que têm demonstrado elevada resistência do agente à penicilina $c$ aos derivados B lactámicos. A resistência do $R$. equi a este grupo de drogas (natural ou adquirida no decorrer da terapia), a baixa penetração celular ou no foco piogranulomatoso, e a dificuldade de aplicação e manutenção da terapia por período prolongado com a penicilina $G$ (via intravenosa) ou a penicilina benzatina (via

\section{restringem}

a

indicação dos derivados 8 lactâmicos na rodococose em potros, ou sugerem que os mesmos sejam utilizados em associação com outros antimícrobianos (Prescott. 1991).

Considerando que, a josamicina foi preconizada originalmente para outras espécies, não foi possível obter na literatura especializada consultada, dados que permitissem a avaliação de efetividade da droga frente às cepas de $R$. equi, comparativamente a outros antimicrobianos comumente utilizados na espécie eqüina.

No presente estudo, a gentamícina e a neornicina apresentaram, respectivamente, efetividade igual ou levemente superior à josamicina. Apesar deste resultado, constatou-se cepas resistentes a estes aminoglicosideos, ao contrário dajosamicina (Tabela 1). Ainda que estas drogas tenham apresentado alta similaridade de ação frente às cepas de $R$. equi, o desenvolvimento de nefrotoxidade após a administração prolongada de aminoglicosídeos (como a gentamícina e neomicina), a reduzida absorção oral da neomicina (Tavares, 1996b) e a ocorrência de resistência das cepas estudadas 
frente à gentamicina e a neomicina (Tabela 1 ), tendem a limitar a indicação dos aminoglicosídeos como alternativos na terapia da rodococose. comparativamente á josamicina.

Embora as cepas de $R$. equi tenham demonstrado alta sensbilidade a eritromicina no presente ensaio in vitro (Tabela 1), e este antimicrobiano seja considerado de eleição para a associação com a rifampicina na terapia da rodococose em potros (Prescott, 1991; Pronost et al., 1993), o uso prolongado deste macrolídeo. por via oral, tem sido associado à complicações orgânicas, incluindo anorexia, cólicas leves, diarréia e úlcera gástrica (Prescott \& Hoffmen, 1993). Adicionalmente, o uso da eritromicina por via intramuscular, pode ocasionar inflamações no ponto de aplicação (Hillidge, 1986), além da ocorrência de cepas resistentes no decorrer da terapia (Prescott\& Sweeney, 1985). A josamicina também possui apresentação para administração via oral, com alta absorção intestinal e sem sinais de toxicidade quando prescrita por período prolongado na terapia de afecções pulmonares em outras espécies (Virbac, 1999). Estas características da droga sugerem a possibilidade do emprego da josamicina, em potros, como alternativa terapêutica pana animais com reações adversas à eritrornicina.

Os casos de pneumonia em potros por $R$. equi, ocorrem em sua maioria, ate o sexto mês de idade, com alta freqüência entre o segundo e terceiro meses, que coincide com o período de transição de imunidade passiva, adquirida pelo colostro, e o início da imunidade ativa efetiva do animal contra o agente (Pronost et et., 1998). Neste sentido, c aumento da quimiotaxia de neutrófilos, macrófagos e células NK em animais tratados com a josamicina (Virbac, 1999), poderia contribuir na resposta imune de potros com rodococose, atuando como complementar á ação antimicrobiana da droga. Adicionalmente, a alta concentração pulmonar da josamicina, associada a capacidade de penetração intracelular da droga em material caseoso (Virbac, 1999), poderiam favorecer a resolução de processos pulmonares desencadeados pelo $R$. equi, caracterizados pela alta refratanedade á terapia antimicrobiana convencional.

Apesar da josamicina ter sido originalmente desenvolvida para a terapia de patógenos respiratórios de suínos e aves, a alta concentração terapêutica pulmonarda droga, a estimulação do sistema imune e a facilidade da prescrição para terapia via oral. são características que favoreceriam a ação deste antimicrobiano em infecções pulmonares por $R$. oqui em potros. Infere-se no presente estudo, que a alta efetividade da josamicina nas cepas estudadas de $R$. equi., aliada a ausência de resistência do agente à droga, permitem sugerir este antimiciobiano como provável alternativa na terapia deste patógeno de relevante importância em sanidade eqüina e no contexto de saúde pública.

RIBEIRO, M. C: CARVALHO FILHO. A. S. DE; MEGID, J,; LISTONI, F. J. P.; DIAS JÚNIOR, J. G. In vitro effect of josamycin in strains of Rhodococcus equi isolated from pulmonar infections in foals. Semina: CL Agrárias, Londrina, v. 21, n. 1, p. 3-8, mar. 2000.

\begin{abstract}
Antmicrobial susceptibility test was performed in thirty-one samples of Rhodococcus equi isolated from iung infections in foals. Among the antimicrobial tosted, erythromycin (100,0 \%), rifampin (96.3\%). neomycin (93,6 \%)Josamycin (90,4\%) and gentamicin (90,4\%)presented the highest sensitivity against $R$ - equi- The most-common occurrence of resistance was observed from cephalexim (100,0 \% lincomycin (100,0\%), cephalothin (96,3\%). oxacillin (96,8\%), penicillin G (96,8\%), amoxicillin (90,3\%) and trimethoprim/sulfamethoxazole (83,8 \%). The highest susceptibility of the R. equi tojosamycin, suggest the drug as alternative for therapy of $R$. equi infections in foals.
\end{abstract}

KEY WORDS: Rhodococcus equi, microbial susceptibility, treatment.

\title{
AGRADECIMENTOS
}

A Profa, Dra. Agueda Castagna de Vargas do Laboratório de Bacteriologia da Universidade Federal de Santa Maria-RS, e a Coleção de Culturas do Instituto Adolfo Lutz de São Paulo-SP, pela cessão de cepas de Rhodococcus. equi isoladas de afecções pulmonares em potros.

Ao Instituto de Biociências da UNESP - Botucatu-SP, pela cessão das cepas controle de Rhodococcus equi (ATCC 33.701-e ATCC 33.701+). 


\section{REFERÊNCIAS BIBLIOGRÁFICAS}

BARTON. M.D.; HUGHES. K.L. Corynebacterium equi: a review. Vet. Bull., v. 50, p. 65-30. 1980.

BAUER, A.V.; KIRBY, W.M.M.; SHERRIS. J. C.; TURCK, M.; Antibiotic susceplity testing by a standardized single disk method. Am. J. Clin. Pathol., v. 45. p. 493-496. 1996.

CORRÊA. W.M.; CORRÊA, C. N.M. Gênero Corynebacteruim. In: Enfermidades infecciosas dos Mamíferos Domésticos. 2. ed.; Rio de Janeiro: Medsi. 1993. p. 133-137-

DOIG. C.; GILL. M.J.; CHURCH.D.L. Rhodococcus equi-an easily missed oppurtunistic pathogen. Scaned. J. Infect. Dis. v. 23. p. 1-6. 1991.

DONISI, A.; SUARDI. M.C.; CASARI. S.; LONGO, M.; CADEO. G.P.; CAROSI. G. Rhodococcus equi infectin in HIVinfectied patients. AIDS, v. 10. p. 359-62. 1996.

DRANCOURT, M.; BONNET. E.; GALLAJS, H.; PELOUX, Y.; RAOULT. D. Rhodococcus equi infcctions in patients with AIDS. J. INFOCT. V. 24 , p. 123-131, 1992-

GAY, G.C.; SLOSS. V.; WRIGLEY, R.H.: HORSEY, R. The treatment of pneumonia in foals caused by Rhodococcus (Corynebacterium) equi. Austr. Vet. J., v. 57. p. 150-161, 1931

GIGUÉRE. S.; PRESCOTT, J.R Clinical manifestations. diagnosis, treatment. and prevention of Rhodococcus equi infections in foals. Vet. Microbiol. v. 56, p. 313-334, 1997.

HAHVEY, R.L.; SUNSTRUN. J.C.Rhodococcus equi infection in patients with and without human immunodeficiency virus infection. Rev, Infect. Dis., v. 13. p. 139-145. 1991.

HILLIDGE. C.J. Review of Corynebcterium (Rhodococcus) equi lung abscesses in foals: Palhogenesis.diagnosis and treatment. Vet. Rec, v. 13, p. 261-264. 1936.

HILLIDGE, C.J. Use of erythromycin-rifampicim combinalion in treatment of Rhodococcus equi pneumonia Vet. Micróbiol., v. 14, p. 337-342, 1937.

HINES.S.A.; KANALY S.T.; BYRNE. B.A.; PALMER,G.H. Immunity to Rhodococcus equi. Vet. Micróbiol., v. 56. p. 177-135. 1997-

LANGONI. H. Rodococose. Uma maneira diferente de falar depneumoniaempotroscausadapelo Rhodococcus equi. Hippus. p. 57-59, fev. 1994.

LINDER. R. Rhodococcus equi and Corynebacterium haemolyticum. Two "Coryneform" bactéria increasingly recognized as agents of human infection. Emerg. Infect. Dis., v. 3. p. 1-10, 1997.

MACCOWAN. K.L.; MANGAWO, M.F. Infections On Rhodococcus equi in children. Diagn. Micrabiol. infect. Dis., v. 14. p. 347-352, 1991.
MOSSER. D.M.; HONDALUS. M.K. Rhodococcus; equi an emerging opportunistic palhogen. Trends Microbiol. v. 4. p. 29-33. 1996

NORDMANN, P.; KERESTEDJAN, J. J.; RONCO. E. Theraphy of Rhodococcus equi disseminated infections in mude mice. Antimicr. Agents Chemother.v. 36, p. 1244-1248, 1992.

PRESCOTT, J.F. Rhodococcus equi: an Animal and Human Pathogen. Clinical Microbiol. REV. v. 4. p. 20-34. 1991.

PRESCOTT. J.R; HOFFMAN. A.M. Rhodococcus equi. Vet. Clin. North Am. Eqüino Pract. v.9, p. 376-384. 1993.

PRESCOTT, J.R,;NICHOLSON.V.M. The effect of combinations of selected antibiotics on the growth of Corynebacterium equi. J. Vet. Pharmacol. Therap. v. 7, p. 61-64. 1984.

PRESCOTT. J.F.; \$WEENEY, C. R. Treatment of Corynebacteruim equi pneumonia; in foals: a review. J. Am. Vet. Med. Ass., v. 137, p. 725-727, 1985.

PRONOST, S.; FORTIER, G.; LEGENDRE. M.F.; OGAWA, K.; SASAKI. Y.; COLLOBERT. C.; TAOUJI, S.; TAKAI, S. Rhodococcus equine: nouvelles approches et bilan de trois anées d'études au laboratoire. Prat. Vét. Equine, v. 30. p. $57-65, \quad 1998$.

SPARKS, S.E.; JCNES, R.L.: KILGORE, W.R. In vitro susceptibility of bacteria to ticarcillin-clavutonic acid combination. Am. J. Vet. Res. v. 49, p. 203S-2040. 1998.

SWEENEY, O.R.; SWEENEY, R.W.; DIVERS. T.J. Rhodococcus equi pneumonia in 48 foals: Response to antimicrobial theraphy. Vet. Microbiol. v. 14, p. 323-336, 1987.

TAVARES, W. Macrolídeos, Lincossamidas e Estreplograminas. In; MANUAL dé Antibióticos e Quimioterápicos Antiinfecciosos, 2. ed. São Paulo: Atheneu, 1996a. p. 481 .

TAVARES, W. Aminociclitóis Aminoglicosídeos- In: MANUAL de Antibióticos e Quimiotorápicos Antiinfecciosos. 2. ed. SãoPaulo; Atheneu, 1996b. p. 408-412.

VIRBAC. Alplucine', $O$ antibiótico imuno-estimulante para melhores performances Manual Técnico. (Departamento Técnico). Sao Paulo. 1999. $18 \mathrm{p}$.

VIVRETTE. S. The diagnosis, treatment, and prevention of Rhodococcus equi pneumonia in foals. Vet. Med., v. 87 , p, $144-140,1992$.

WILSON, W.O. Foal pneumonia. In: CURRENT Theraphy in Equine Medicine. 3. ed-Philadelphia: W.B. Saunders. 1992. p. $466-473$.

WOOLCOCK. J.B.; MULTIMER, M, D. Corynebacterium equi: in vitro susceptitbility to twenty-six antimicrobial agents. Antimicr. Agents Chemother., v. 13, p. 976-977. 1980. 